\title{
Mariano Artigas (1938-2006). Testimonio de unos encuentros y breves reflexiones sobre el Grupo de Investigación Ciencia, Razón y Fe (CRYF) de la Universidad de Navarra
}

\author{
Mariano Artigas (1938-2006). Testimony of some \\ meetings and brief reflections on the Research Group \\ Science, Reason and Faith (CRYF) of the University \\ of Navarra
}

\section{JOSÉ MANUEL GIMÉNEZ-AMAYA*}

Universidad de Navarra

jmgimenezamaya@unav.es

No es tarea fácil compaginar el rigor científico con la profundidad filosófica y teológica. El Prof. Artigas posee una reconocida capacidad de los tres ámbitos, y en su nuevo libro consigue un resultado excelente. Estudia con seriedad los interrogantes que surgen a propósito del hombre, considera los datos científicos actuales, y con un lenguaje que compagina la amenidad con el rigor, muestra los caminos que permiten comprender la dignidad de la persona humana y proporciona bases firmes para la reflexión ética. (Portillo 1992, 6) ${ }^{1}$.

Profesor Ordinario de la Facultad Eclesiástica de Filosofía y miembro del Grupo de Investigación Ciencia, Razón y Fe (CRyF) de la Universidad de Navarra. Ha sido director del CRYF desde el año 2010 al 2016. El autor agradece las críticas y sugerencias de los profesores Sergio Sánchez-Migallón y Santiago Collado de la Universidad de Navarra.

1 Palabras del entonces Gran Canciller de la Universidad de Navarra en el prólogo al libro del profesor Mariano Artigas, El hombre a la luz de la ciencia $(1992,6)$. 
So we only know how to direct our enquiries now, if we have first made our own the philosophical thought of our predecessors. (MacIntyre 2009, 1) $)^{2}$.

Resumen. En esta contribución se narra el contacto del autor con el profesor Mariano Artigas y, especialmente, con el Grupo de Investigación Ciencia, Razón y Fe (CRYF) que él fundó en 2003 en la Universidad de Navarra. También se hacen unas breves reflexiones sobre la importancia de los trabajos del CRYF en el contexto de la labor universitaria.

Palabras clave: Mariano Artigas; Grupo CRYF; ciencia y fe; universidad.

Abstract. This contribution is aimed to emphasise the author's personal contact with Professor Mariano Artigas and, especially, with the Research Group in Science, Reason and Faith (CRYF, in Spanish) which he founded in 2003 at the University of Navarre. In addition, some reflections about the importance of this Research Group in the context of the development of the university studies are pointed out.

Keywords: Mariano Artigas; CRYF Group; science and faith; university.

\section{Relato de mis encuentros con Mariano Artigas}

Mi contacto personal en vida con el profesor Mariano Artigas, se reduce a una brevísima conversación telefónica que mantuvimos cuando los dos trabajábamos en la Universidad de Navarra en la década de los 90 del siglo pasado. Aunque ahora mi recuerdo es algo borroso, nuestro breve coloquio se desarrolló en Pamplona, siendo yo director del Departamento de Anatomía de la Facultad de Medicina y él profesor de la Facultad Eclesiástica de Filosofía. El motivo de dicha conversación era una interpretación que yo había hecho de algunas palabras o reflexiones del profesor Artigas acerca del conocido neurocientífico Ramón y Cajal y de la neurociencia. La conversación fue muy breve porque me hizo ver que él no afirmaba la opinión que yo le atribuía. Todo quedó en un malentendido por mi parte ${ }^{3}$.

2 "De esta manera, solamente sabremos como dirigir nuestras investigaciones hoy en día, si hemos hecho propio el pensamiento filosófico de nuestros antepasados”. La traducción al castellano es nuestra.

3 Pienso que bien podría haber sido con relación a uno de los siguientes escritos de Mariano Artigas: "Buscando el alma con el bisturí. El Dr. Crick y su cerebro" o "El término de una búsqueda sin término. Karl Popper dialoga con la muerte”, ambos publicados en 1994. Sin embargo, en ninguno de ellos se menciona a Ramón y Cajal, por lo que no tengo seguridad ahora a este respecto. 
La siguiente oportunidad que tuve relación con D. Mariano fue en diciembre de 2006. Por aquellas fechas yo viajaba con frecuencia a Pamplona desde Madrid, y, cuando estaba en Pamplona, me alojaba en el Colegio Mayor Belagua del campus universitario. Allí asistí a su funeral corpore in sepulto. Recuerdo que me impresionó hondamente la homilía que el vicario del Opus Dei de la delegación de Pamplona, D. José Manuel Martínez, pronunció en esa ocasión. Me llamó mucho la atención que al mencionar sus publicaciones, se hiciera referencia a una reciente, publicada en la prestigiosa editorial universitaria Oxford University Press, que él había finalizado muy poco antes de su fallecimiento ${ }^{4}$.

A partir de ese momento, me fui acercando más y más a su persona. Especialmente a través de los profesores del Grupo de Investigación Ciencia, Razón y Fe (CRYF), que él había fundado junto a otros investigadores de la Universidad de Navarra 5 .

A finales del año 2007 fui invitado a impartir un seminario al CRYF por el profesor Santiago Collado, en el que hablé sobre algunos aspectos de la neurociencia y su relación con la libertad. ${ }^{6}$. A partir de ese contacto más formal, y siempre siguiendo el consejo y asesoramiento del profesor Sergio

4 Se trataba de Oracles of Science. Celebrity Scientist versus God and Religion, escrito junto con Karl Giberson. Poco antes de fallecer en Pamplona, el profesor Santiago Collado le llevó al hospital las pruebas de imprenta de este libro y el profesor Artigas cambió el orden de los autores, colocando primero a Giberson ya que él se iba a morir pronto -señalóy el autor canadiense sería quien tendría que defender el libro. El profesor Mariano Artigas había publicado también otros dos libros con una difusión internacional de especial relevancia: Mind of the Universe. Understanding Science and Religion, Templeton Press, West Conshohocken 2001 y, con W. R. Shea, Galileo in Rome. The Rise and Fall of a Troublesome Genius.

5 Los profesores que, junto a Mariano Artigas, integraron el CRYF en su inicio fueron: Santiago Collado (Facultad Eclesiástica de Filosofía), Francisco Gallardo (Facultad Eclesiástica de Filosofía), Juan Luis Lorda (Facultad de Teología), Antonio Pardo (Facultad de Medicina) y Carlos Pérez (Facultad de Ciencias), que falleció en un accidente de montaña en julio de 2005. Este grupo de investigación de la Universidad de Navarra se reunió por primera vez el 5 de marzo de 2003. Actuó como secretario del CRYF el profesor Santiago Collado.

6 El seminario se impartió el martes 18 de diciembre de 2007 en el aula 1 del edificio de las Facultades Eclesiásticas de la Universidad de Navarra con el título "Una aproximación interdisciplinar a la relación entre neurociencia y libertad”. Un resumen y fotografías de este seminario pueden verse en la web del CRYF (www.unav.es/cryf). 
Sánchez-Migallón, me fui acercando más y más al CRYF, asistiendo con más frecuencia a sus reuniones y seminarios. En esa época el director del CRYF era el profesor Héctor Mancini ${ }^{7}$, de la Facultad de Ciencias, y el secretario el profesor Santiago Collado.

Yo llevaba ya algún tiempo estudiando filosofía, con objeto de intentar fundamentar más sólidamente mis actividades interdisciplinares. Y como ya he señalado también iba asistiendo regularmente a las reuniones del CRYF en Pamplona, viajando desde Madrid, donde ejercía como catedrático de Anatomía y Embriología de la Facultad de Medicina de la Universidad Autónoma de Madrid. Fue entonces cuando se produjo mi tercer "encuentro" con Mariano Artigas.

En los primeros meses de ese mismo año, me había incorporado ya como miembro socio del CRYF ${ }^{8}$. En ese tiempo, el profesor Santiago Collado también me había sugerido la posibilidad de asistir a una reunión científica que organizaba el CRYF en Londres, en colaboración con el Thomas More Institute, para celebrar un homenaje al profesor Artigas. Fue durante los días 7-9 de mayo del año 2009. En ese foro impartí una conferencia ${ }^{9}$ y tuve ocasión de asistir a otras, de importantes investigadores, sobre las relaciones entre ciencia y religión. Me impresionó mucho el reconocido prestigio que daban a Mariano Artigas en este campo del saber ${ }^{10}$.

Durante esa estancia en Inglaterra que acabo de mencionar, pude asistir también al funeral de Sir John Templeton ${ }^{11}$ en la Westminster Abbey de

$7 \quad$ El profesor Héctor Mancini (profesor Ordinario de Física en la Facultad de Ciencias de la Universidad de Navarra) fue elegido director del CRYF en el año 2007 para sustituir al profesor Mariano Artigas, después del fallecimiento de este último. Continuó como director del CRYF hasta enero de 2010 cuando le sustituí yo.

8 Concretamente el 24 de febrero de 2009, junto con el profesor Luis Echarte de la Facultad de Medicina de la Universidad de Navarra.

9 El título fue "Understanding of free will: a philosophical and neuroscientific approach", que puede verse también en nuestra página web.

10 Puede consultarse el libro que recoge mucho de lo que se dijo en esa reunión: Science and Faith within Reason, editado por J. Navarro.

11 Mariano Artigas había conocido personalmente a Sir John Templeton y había recibido ayudas de su Fundación para realizar estudios sobre ciencia y religión. Concretamente con una de ellas escribió el libro Mind of the Universe ya mencionado en este escrito. Cuando falleció el profesor Artigas estaba en curso otra ayuda que se completó bajo la dirección 
Londres junto con el profesor Santiago Collado -los dos representábamos al CRYF-y el profesor Sergio Sánchez-Migallón, vicedecano de la Facultad Eclesiástica de Filosofía, que representaba la institución universitaria de la que Mariano Artigas había sido decano ${ }^{12}$. Nunca olvidaré el cordial saludo que nos dirigió a los tres el Dr. Jack Templeton -hijo de Sir John Templeton, presidente entonces de la Fundación Templeton hasta su fallecimiento en mayo de 2015- al recordarle al profesor Mariano Artigas.

En enero de 2010 fui elegido director del CRYF por un periodo de tres años, que se me renovó en 2013 para un segundo mandato. En enero de 2016 dejé este cargo, y me sustituyó el profesor Javier Sánchez Cañizares. En estos años de intenso contacto con el CRYF, especialmente como director del grupo, es donde pienso que se ha producido el definitivo y más cercano encuentro con Mariano Artigas, que se ha hecho para mí una figura muy próxima. Y esto por tres motivos conceptuales.

En primer lugar, por la relevancia para el mundo actual de las investigaciones que se desarrollan desde el CRYF. En segundo término, por el reto al conocimiento que supone el trabajo verdaderamente interdisciplinar, y que hace de su logro un verdadero servicio. Y, finalmente, por la importante contribución de estos estudios en una universidad y en una sociedad que están cambiando muy rápidamente.

En el resto de este escrito quiero apuntar, con brevedad, algunas ideas sobre estos tres últimos aspectos mencionados.

\section{Relevancia de los estudios sobre Ciencia, Razón y Fe}

La situación actual de las relaciones entre la ciencia y la religión hacen que grupos como el CRYF sean de una gran ayuda para la investigación interdisciplinar y la docencia en las instituciones educativas. Estas relaciones se ven con creciente interés pero, al mismo tiempo, se experimentan también

del profesor Santiago Collado.

12 El profesor Mariano Artigas fue decano de la Facultad Eclesiástica de Filosofía de la Universidad de Navarra desde el curso 1988-1989 hasta el curso 1997-1998. 
como problemáticas (Giménez 2013) o, al menos, como enfoques paralelos, en pocos casos como netamente convergentes.

En el origen de esta visión "dicotómica” de la realidad estudiada por la ciencia y la religión está, en gran medida, la instauración del fenómeno filosófico que se ha conocido como "modernidad". No es el objetivo de estas breves consideraciones hacer un análisis pormenorizado de este rasgo tan complejo y tan influyente en la cultura y el pensamiento ${ }^{13}$. Nuestra pretensión, en este apartado, es apuntar brevemente dos aspectos -dentro de un análisis restringido a la antropología filosófica- que se han ido desarrollando en los últimos siglos y que nos parecen relevantes para analizar este "hiato" producido entre la ciencia y la religión.

El primero de ellos tiene que ver con el desarrollo de la propia disciplina, la antropología filosófica. La crisis del pensamiento moderno es, en gran medida, una crisis del conocimiento. Un conocimiento que se refiere al sujeto como el gran protagonista -y, progresivamente, casi único-de ese magno empeño gnoseológico. Pues bien, la modernidad se puede caracterizar, precisamente, por la afirmación y descomposición en el ser humano de su propia subjetividad ${ }^{14}$.

Me explicaré mejor. Con el fenómeno de la modernidad se produce también el nacimiento de la ciencia moderna. Esto último se enmarca en la valoración de la experiencia y un creciente optimismo de base sobre las posibilidades del ser humano de conocer la realidad que se muestra a los sentidos. Ese optimismo gnoseológico también va a ir acompañándose de un "contrapeso" negativo, que se podría enunciar como "las dudas heredadas acerca del hombre para conocer a Dios y con el carácter arbitrario de la voluntad divina y humana, que arroja sus sombras sobre el cosmos y la condición humana" (Murillo 2006, 36).

13 La bibliografía es extensísima. Remito al lector interesado a los siguientes trabajos generales, y la bibliografía referida en ellos: Rodríguez 2000, 9-19, Valverde 2003, Taylor 2007, Giménez-Amaya, y Sánchez-Migallón 2011. Para ver este fenómeno en un contexto más teológico, puede ser útil consultar, entre otras muchas, las siguientes referencias: Guardini, 1995, Müller, 1998, 205-209; Ratzinger, y Habermas 2006 y Kasper 2013, 21-32.

14 Nos hemos guiado aquí por las interesantes reflexiones de José Ignacio Murillo (2006). 
A partir de aquí sería pertinente desarrollar pormenorizadamente un itinerario que abarca el nominalismo y sus presupuestos gnoseológicos, el dualismo cartesiano, Kant, Hegel, el idealismo alemán, el materialismo marxista y sus corolarios, todo ello desembocando en la filosofía del siglo $\mathrm{XX}^{15}$. De forma muy sumaria, en este camino del pensamiento cultural-antropológico moderno va quedando patente la creciente dificultad para entender y defender la realidad del espíritu, "que comienza a aparecer como un espejismo que cabe explicar y reducir a leyes históricas y materiales" (Murillo 2006, 38) ${ }^{16}$. Además, se abandona la explicación del hombre basada en su naturaleza, y su estudio se desplaza hacia las ciencias históricas (Murillo 2006, 38).

Finalmente, convendría añadir que el marco conceptual de la modernidad se ha visto ampliado por la presencia, cada vez más notoria, del pensamiento posmoderno. Según el filósofo moral de origen británico Alasdair MacIntyre, el primero en detectar con lucidez el fracaso del proyecto moderno es Nietzsche, aunque su actitud ante este descubrimiento fue una huída hacia adelante, propugnando la autoafirmación del "superhombre” ante el vacío de normas morales de la propia modernidad; en esta autoafirmación se encuentran también una de las principales raíces del pensamiento

15 También puede ser interesante consultar la entrevista al profesor Alejandro Llano (2011, 333-396); y el original enfoque del profesor Juan Bautista Fuentes (2009, 95-144).

16 También me parece relevante dejar aquí constancia de unas palabras de Henri de Lubac (Lubac 1985, 78-79): “Algunos sitúan su comienzo en el 'siglo de las luces'; otros hacen retroceder un poco su aparición hasta las críticas de Kant ; para otros su gran iniciador es Hegel [...]. Hay también quienes sostienen que comienza con el espíritu científico “positivista” [...] o con la aplicación exclusiva del espíritu científico al estudio del hombre, tomado como objeto del laboratorio [...]. En una palabra, una vez más, con la repulsa de toda reflexión metafísica, así como de toda religión. Tal sería la última y definitiva conquista: negarse a ver en el hombre aspiración alguna trascendente [...]. Se puede utilizar [la palabra "modernidad"] como vector de una cierta actitud general adoptada por un buen número de intelectuales, bajo el impacto de las extraordinarias conquistas de la ciencia moderna y de las no menos profundas desilusiones en que han venido a resolverse los grandes sueños del progreso y de la autodeificación del hombre. En este caso podría decirse que el origen primero de la "modernidad", su espíritu profundo [...] es el rechazo de toda fe, consecuencia del rechazo del misterio humano” (Cf. Romera 2007, 111-137). 
posmoderno. El propio MacIntyre (1987, 141-154) entiende su propuesta como una alternativa a la de Nietzsche ${ }^{17}$.

Todo, en definitiva, lleva a una fractura en el estudio del ser humano que incapacita para llegar a un verdadero conocimiento del mismo y de su actuar $^{18}$.

Un segundo aspecto al que me querría referir aquí se relaciona con el crecimiento formidable en los últimos siglos de las ciencias biológicas y médicas, que ha puesto sobre la mesa la importancia de entender la biología humana en el contexto de un ser vivo -el ser humano-que es más que pura biología, y entender como esta se articula con su racionalidad. En efecto, la modernidad ha favorecido una respuesta materialista o naturalista que ha sido como el colofón de un pensamiento que no "puede" llegar a más, porque, sencillamente, no encuentra la manera de integrar un enfoque del hombre que haga justicia a todo lo que es en realidad. Se ha ido perdiendo, a lo largo del camino de los siglos, un enfoque verdaderamente "holístico" del ser humano ${ }^{19}$.

La respuesta de varios autores, formados en el ámbito de la fenomenología, con una aproximación antropológica "vitalista" ha sido de gran interés para exponer con claridad esta falacia de un ser humano donde la última palabra la dice "exclusivamente" su biología. Es mérito de este grupo de filósofos el intento de conciliación y coordinación de la visión biológica

17 El capítulo lleva el sugerente título de "Nietzsche o Aristóteles".

18 Como se ha indicado ya para muchos investigadores, el núcleo central del problema de la modernidad es estrictamente gnoseológico y se establece con la pregunta ¿qué podemos conocer? Las consecuencias que se siguen de la respuesta que se de a esa cuestión afectan, sobre todo, a la visión antropológica y ética que se tiene del hombre. Sin embargo, una de las grandes preguntas que es preciso también plantearse en este contexto es si es perjudicial todo el proyecto ilustrado de la modernidad (Cf. Giménez 2011, 180-183). En este trabajo no pretendemos entrar en el núcleo de la cuestión, pero tampoco dejar de mencionarlo. Así, por ejemplo, Benedicto XVI $(2010,69)$ dijo textualmente: "Lo importante es que intentemos vivir y pensar el cristianismo de tal manera que asuma en sí la buena, la correcta modernidad, y que al mismo tiempo se aparte y distinga de lo que se ha convertido en una contrarreligión”. (Cfr. Giménez 2013).

19 Quizá sea útil aquí recordar las conocidas palabras del gran poeta Thomas Eliot en Opening Stanza from Choruses (1934): "Where is the Life we have lost in living? / Where is the wisdom we have lost in knowledge? / Where is the knowledge we have lost in information?". 
del ser humano con los datos aportados con las ciencias humanas. Max Scheler refleja muy bien esta inquietud y esta aspiración, cuando dice en 1928 "tenemos una antropología científico-natural, otra filosófica y otra teológica indiferentes entre sí, pero no tenemos una idea unitaria del hombre" (2000, 3).

Concluyo este apartado como lo inicié. Pienso que grupos como el CRYF son muy necesarios para intentar ofrecer esta visión "global” de la vida y el actuar humanos. Este ha sido mi interés investigador desde mi incorporación a este grupo de la Universidad de Navarra en $2009^{20}$.

\section{El "servicio" interdisciplinar}

La palabra "interdisciplinaridad" ha sido utilizada mucho y en contextos diversos. Muchas veces, en nuestra opinión, sin todo el sentido profundo y sapiencial que requiere. En este apartado queremos hacer dos breves consideraciones al hilo del ámbito para el que se escriben estas consideraciones.

En primer lugar, un comentario general pero que me parece importante señalar como preámbulo. La interdisciplinaridad representa un "servicio" en el ámbito de los estudios de ciencia y religión. Esta actitud de servicio se debe poner de manifiesto con claridad en el esfuerzo que hay que hacer para profundizar en campos nuevos, para entender perspectivas diferentes, para estudiar conceptos diversos. El tiempo para el estudio y el trabajo no es indefinido. Exige selección y sacrificio para aventurarse en estos campos interdisciplinares, a expensas de una dedicación más exclusiva a temas muy especializados. Además, aunque es cierto que esta actitud interdisciplinar es muy enriquecedora, requiere también una buena dosis de paciencia y fuerza de voluntad para descubrir sus frutos tanto en la docencia como en la investigación. En la propia sociedad y en la universidad en la que vivimos

20 En estos años en el CRYF, en colaboración con otros profesores de la Universidad de Navarra y de la Santa Cruz de Roma, he publicado los siguientes libros relacionados con estudios interdisciplinares sobre ética y antropología: De la neurociencia a la neuroética. Narrativa científica y reflexión filosófica con S. Sánchez-Migallón, y con J. A. Lombo La unidad de la persona. Aproximación antropológica desde la filosofía y la neurociencia y Biología y racionalidad. Lo específico humano en el plano orgánico. 
no se valoran suficientemente estos estudios, pero, irónicamente, son muy necesarios para comprender el propio mundo en que vivimos.

En segundo término, me gustaría resaltar una visión de la interdisciplinaridad desde dos puntos de vista que pueden proporcionar fecundidad a este tipo de trabajos. Estos dos sentidos se resumen en ver la interdisciplinariedad desde la perspectiva metodológica y desde un horizonte propiamente resolutivo o terapéutico.

Decir que desde la perspectiva metodológica es de gran importancia el diálogo y la colaboración entre las distintas disciplinas del saber -especialmente entre las distintas subdisciplinas experimentales- es casi un lugar común, después de los avances en el mundo de la ciencia experimental que hemos comprobado en estos últimos siglos ${ }^{21}$. Pero, ¿esta interdisciplinaridad es realmente capaz de borrar fronteras y límites, para aunar conocimientos auténticamente sapienciales, que aborden los problemas de conocimiento subyacentes a las preguntas radicales sobre el saber y el actuar humanos? ¿Es terapéuticamente competente para restañar esta división (y disgregación) del saber que ha resultado de la modernidad?

Todo hace pensar que sí. La propia idea de fragmentación en el saber, que se aventura como uno de los defectos nucleares de su transmisión en la modernidad, reclama, casi de forma natural, la recuperación de una integración del conocimiento ${ }^{22}$. Esta interdisciplinaridad, sin embargo, debe abrirse también a la filosofía y a la teología ${ }^{23}$; y, estas últimas, ocupando

21 El breve artículo de Rosell, de las Heras, y Giménez (1998), dedicado a la importancia de la interdisciplinaridad en el desarrollo de la neurociencia moderna, puede ser interesante también en relación con la visión interdisciplinar en general.

22 Un muy buen resumen de estas ideas, son las palabras que pronunció Mons. Javier Echevarría, Gran Canciller de la Universidad de Navarra, en 2011: "La educación universitaria ha de forjarse sobre una visión amplia y profunda del ser humano. Esta antropología abierta a la trascendencia requiere la aportación de las diversas ciencias, con especial énfasis en los saberes humanísticos. El cultivo y la docencia de la teología y de la filosofía garantizan que el humanismo universitario no se reduzca a una agregación superficial de conocimientos, sino que aspire a lograr una visión equilibrada y completa de la persona".

23 La bibliografía es también muy amplia. Menciono aquí solo dos pasajes que me parece podrían ser relevantes en el contexto de este escrito. El primero es de Benedicto XVI (2006): “Con todo, como he tratado de demostrar, la razón moderna propia de las ciencias 
un centro geográfico en el pensamiento y en la formación intelectual, son capaces de proporcionar unidad a las distintas ciencias entre sí.

\section{Una contribución profunda a la Universidad}

Nos enfrentamos, por tanto, a un serio problema de fragmentación del saber. La falta de interconexión de las ciencias entre sí y la necesidad de especialización cada vez más acuciante de los últimos tiempos ha dejado un poso amargo de ausencia de síntesis y entendimiento integrativo (Giménez 2012). Un lugar privilegiado para observar estos fenómenos es la universidad que vivimos en la actualidad.

Como ha señalado el profesor Lluís Clavell, la fragmentación en los conocimientos arroja este balance: "un mosaico de visiones fragmentarias de la realidad -en su mayoría del mundo de las ciencias y no de las humanidades - que no facilitan que la persona, que es un todo unitario, pueda orientarse en orden a su realización y perfeccionamiento. De este modo, la síntesis, en quienes de algún modo la realizan, queda en la mayor parte de los casos a merced de una valoración subjetiva” (1992, 600).

Para la reconstrucción de esta fragmentación del saber, la interdisciplinariedad se abre como un camino muy a tener en cuenta en el seno de la actividad universitaria. En efecto, una propuesta interesante para promover esta interdisciplinariedad podría ser intentar elaborar un pensamiento sapiencial en la propia materia en la que se es especialista. Es, sin lugar

naturales, con su elemento platónico intrínseco, conlleva un interrogante que va más allá de sí misma y que trasciende las posibilidades de su método. La razón científica moderna ha de aceptar simplemente la estructura racional de la materia y la correspondencia entre nuestro espíritu y las estructuras racionales que actúan en la naturaleza como un dato de hecho, en el cual se basa su método. Ahora bien, la pregunta sobre el por qué existe este dato de hecho, la deben plantear las ciencias naturales a otros ámbitos más amplios y altos del pensamiento, como son la filosofía y la teología”. El segundo pasaje es de Hans Urs von Balthasar (1997, 22): "El hombre positivista-ateo de hoy, cegado, no solo para la teología, sino incluso para la filosofía, debía, situado ante el fenómeno de Cristo (el solo resplandor del Dios glorioso y sublime), aprender a 'ver' de nuevo”. 
a dudas, una forma de entendimiento más profundo y significativo del propio saber especializado ${ }^{24}$.

Es muy conocida la referencia a Benito de Nursia, que hace Alasdair MacIntyre al final de su afamado libro Tras la virtud (1981, 322). Para este filósofo es necesario ahora más que nunca esperar a un nuevo san Benito. Siempre se ha visto que estas reflexiones de MacIntyre son una referencia explícita a la importancia de dar más preeminencia a la religión en una etapa tan oscura para el saber y para la vida moral como la que vive el hombre contemporáneo. Sin embargo, el propio autor hace en 2007 un balance de su obra, publicada por primera vez en 1981: “mi intención era sugerir que el nuestro es también un tiempo con nuevas e impredecibles posibilidades de renovación. Es un tiempo para resistir todo lo que sea posible, con prudencia y con coraje, con adecuación y templanza, al orden social, económico y político dominante en la modernidad avanzada. Así era hace 26 años. Y así sigue siendo ahora" (MacIntyre 2007, xiii-xiv).

El profesor David Solomon (2012, 256-257) señala que, para MacIntyre, el santo fundador de Europa representa en realidad una fuerza paradigmática para preservar la tradición clásica y las virtudes en pequeñas comunidades, en una época de ceguera muy general para esos valores. En el contexto de este trabajo es difícil no percibir en la formación universitaria que nos propone MacIntyre, una de esas comunidades que resisten el (aparente) empuje de una sociedad cuyo rumbo moral y vital ha naufragado y donde la fragmentación sapiencial del saber es evidente.

He recordado a Mariano Artigas y uno de los frutos de su trabajo universitario, el CRYF. Pero lo que de verdad queda no es solo un trabajo

24 Cf. Romera (2009, 95-109), este autor sostiene que "El debate interdisciplinar [es útil] en el seno de la universidad, porque [obliga] a enfrentarse con uno mismo y con la propia disciplina, a reconsiderar las propias ideas y quizás a sentir el estímulo de ampliar los horizontes de estudio. Todo ello ayuda a elaborar de un modo riguroso las propias comprensiones intelectuales en el ámbito científico en el que el especialista se mueve, sin descuidar los protocolos de investigación ni hacer caso omiso de los resultados consolidados en la propia ciencia. En último término, la diferencia entre una academia y una universidad estriba en que la primera se limita a enseñar con competencia y eficacia lo que otros han elaborado, mientras que la institución universitaria enseña lo que en ella se ha pensado" (Romera 2009, 100). 
y una disposición interdisciplinar ceñida a los estudios concretos. Lo que ha quedado, sobre todo, son personas que quieren seguir este trabajo. En palabras del fundador de la Universidad de Navarra: "No hay Universidad propiamente en las Escuelas donde, a la transmisión de los saberes, no se une la formación enteriza de las personalidades jóvenes. Ya el humanismo helénico fue consciente de esta riqueza de matices. Pero cuando - llegada la plenitud de los tiempos-Cristo iluminó para siempre las arcanas lejanías de nuestro destino eterno, quedó establecido un orden humano y divino a la vez, en cuyo servicio tiene la Universidad su máxima grandeza” (Escrivá 1993, 77).

\section{Referencias}

Artigas, M. 1992. El hombre a la luz de la ciencia. Madrid: Palabra.

-. 1994a. "El término de una búsqueda sin término. Karl Popper dialoga con la muerte”, Aceprensa, 28 Septiembre.

-. 1994b. "Buscando el alma con el bisturí. El Dr. Crick y su cerebro." Aceprensa 23 Noviembre.

-. 2001. Mind of the Universe. Understanding Science and Religion. West Conshohocken: Templeton Press.

Balthasar, H. U. von. 1997. Teológica. Verdad del mundo. Madrid: Encuentro.

Benedicto XVI. 2006. Fe, razón y universidad. Recuerdos y reflexiones, Discurso en la

Universidad de Ratisbona, Ratisbona, 12 de septiembre.

-. 2010. Luz del mundo. El papa, la iglesia y los signos de los tiempos. Una conversación con Peter Seewald. Barcelona: Herder.

Clavell, L. 1992. "Ética y unidad del saber (Reflexiones para una acción cultural universitaria)." Scripta Theologica 24:595-602.

Echevarría Rodríguez, J. 2011. Discursos pronunciados en la investidura del grado de doctor "honoris causa". Pamplona: Universidad de Navarra, 27 de octubre.

Eliot, T. S. 1934. The Rock. London: Faber \& Faber.

Escrivá de Balaguer, J. 1993. Josemaría Escrivá de Balaguer y la Universidad. Pamplona: Eunsa.

Fuentes, J. B. 2009. La impostura freudiana. Madrid: Encuentro.

Giberson, K., y Artigas, M. 2007. Oracles of Science. Celebrity Scientist versus God and Religion. New York: Oxford University Press. 
Giménez-Amaya, J. M. 2011. La universidad en el proyecto sapiencial de Alasdair MacIntyre. Pamplona: Universidad de Navarra.

-. 2012. “La fragmentación y ‘compartimentalización’ del saber según Alasdair

MacIntyre.” En Sapienza e Libertà. Studi in onore del Prof. Lluís Clavell, editado por M. Pérez de Laborda, 193-202. Roma: Edusc.

-. 2013a. ¿Cómo son las relaciones entre la fe y la ciencia en la actualidad?” En 50 preguntas sobre la fe, editado por J. Miras, y T. Trigo, 69-71. Pamplona: Eunsa. -. 2013b. "Relaciones ciencia y religión en el siglo XXI: ¿fracaso de la llamada "razón secular”? Scientia et Fides 1:53-61.

Giménez-Amaya, J. M., y S. Sánchez-Migallón. 2010. De la neurociencia a la neuroética. Narrativa científica y reflexión filosófica. Pamplona: Eunsa.

-. 2011. Diagnóstico de la universidad en Alasdair MacIntyre. Génesis y desarrollo de un proyecto antropológico. Pamplona: Eunsa.

Guardini, R. 1995. El fin de la modernidad. Quién sabe de Dios conoce al hombre. Madrid: PPC.

Kasper, W. 2013. El Evangelio de Jesucristo. Obra completa de Walter Kasper, vol. 5, 21-32. Santander: Sal Terrae.

Llano, A. 2011. Caminos de la filosofía, Pamplona: Eunsa.

Lombo, J. A., y J. M. Giménez-Amaya. 2013. La unidad de la persona. Aproximación antropológica desde la filosofía y la neurociencia. Pamplona: Eunsa.

-. 2016. Biología y racionalidad. El carácter distintivo del cuerpo humano. Pamplona: Eunsa.

Lubac, H. de. 1985. Diálogo sobre el Vaticano II. Recuerdos y reflexiones. Madrid: BAC. MacIntyre, A. 1981. Tras la virtud. Barcelona: Crítica.

-. 2007. After Virtue: A Study in Moral Theory. 3rd ed., Notre Dame: University of Notre Dame Press.

-. 2009. God, Philosophy, Universities. A Selective History of the Catholic Philosophical Tradition. Lanham: Rowman \& Littlefield Publishers, Inc.

Müller, G. L., Dogmática. Teoría y práctica de la teología, Herder, Barcelona 1998.

Murillo, J. I. 2006. “Antropología.” En Diccionario de Teología, editado por C. Izquierdo, J. Burggraf, y F. M. Arocena, 29-49. Pamplona: Eunsa.

Navarro, J., ed. 2011. Science and Faith within Reason. Oxford: Ashgate.

Portillo, A. del, 1992. Prólogo a El hombre a la luz de la ciencia, de M. Artigas. Madrid: Palabra.

Ratzinger, J., y J. Habermas. 2006. Dialéctica de la secularización. Sobre la razón y la religión. Madrid: Encuentro.

Rodríguez Duplá, L. 2000. “Qué rasgos definen la cultura emergente?” En Qué tipo de persona queremos educar para el nuevo milenio, 9-19. Madrid: Bruño. 
Romera, L. 2007. “La religione e le vicende filosofico-culturali del Novecento." En La verità della religione. La specificità cristiana in contesto, editado por G. Tanzella-Nitti, G. Maspero, 111-137. Roma-Siena: Cantagalli.

-. 2009. La actualidad del pensamiento cristiano. Piura: Universidad de Piura.

Rosell, A., S. de las Heras, y J. M. Giménez-Amaya. 1998. "Neurociencia: ejemplo del abordaje multidisciplinar como estrategia eficaz en la investigación científica.” Revista de Neurología 27:1071-1073.

Scheler, M. 2000. El puesto del hombre en el cosmos. Barcelona: Alba.

Shea, W. R., y M. Artigas. 2003. Galileo in Rome. The Rise and Fall of a Troublesome Genius. New York: Oxford University Press.

Solomon, D. 2012. “MacIntyre.” En Ethics: The Key Thinkers, editado por T. Angier. London: Bloomsbury.

Taylor, C. 2007. A Secular Age. Cambridge, Mass.: Harvard University Press.

Valverde, C. 2003. Génesis, estructura y crisis de la modernidad. Madrid: BAC. 